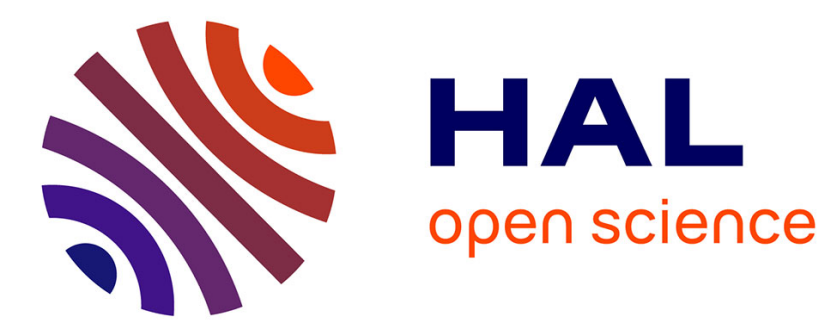

\title{
Pour une anthropologie clinique de la rencontre pédagogique
}

\author{
Françoise Hatchuel
}

\section{To cite this version:}

Françoise Hatchuel. Pour une anthropologie clinique de la rencontre pédagogique. Spirale - Revue de Recherches en Éducation , 2003, 31, pp.91-103. halshs-00354089

\section{HAL Id: halshs-00354089 \\ https://shs.hal.science/halshs-00354089}

Submitted on 13 Dec 2017

HAL is a multi-disciplinary open access archive for the deposit and dissemination of scientific research documents, whether they are published or not. The documents may come from teaching and research institutions in France or abroad, or from public or private research centers.
L'archive ouverte pluridisciplinaire HAL, est destinée au dépôt et à la diffusion de documents scientifiques de niveau recherche, publiés ou non, émanant des établissements d'enseignement et de recherche français ou étrangers, des laboratoires publics ou privés. 


\section{Pour une anthropologie clinique de la rencontre pédagogique}

Spirales, 31 (janvier 2003), pp.91-103

Françoise Hatchuel

CREF-Paris X

Secteur « savoirs et rapport au savoir»

Résumé : à partir de ses différentes recherches autour du rapport au savoir des élèves et des interactions enseignant-e-s/ élèves, l'auteure se centre ici sur la notion de rencontre pédagogique, c'est-à-dire de rencontre, autour d'un savoir à acquérir et à un instant donné, de plusieurs individus porteurs chacun, d'une histoire et d'un vécu qui les amènent à interpréter en termes d'attentes et d'espérances les informations prises chez l'autre. Dans une double perspective de recherche et de formation, elle s'efforce d'une part de repérer quelques indicateurs de la façon dont se négocie cette rencontre, d'autre part de comprendre ce qui pourrait la faciliter.

Abstract : this article is based upon différent research by the author concerning relation with knowledge and interactions between teachers and pupils. It is centred on the notion of pedagogical meeting, that is to say the meeting, with a knowledge to get and in a precise moment, of several people carrying their own experience, which bring them to interpret informations taken from each other in terms of waiting and hope. In a double view of formation and of research, the author tries on one hand to see how this meeting is negociated, and on the other hand to show what could make it easier.

Mots clés: interactions, rencontre pédagogique, perspective anthropologique, approche clinique, autonomie, rapport au savoir.

Keywords : interactions, pedagogical meeting, anthropological view, clinical approach, autonomy, relation with knowledge.

Dans le cadre d'une approche ethnographique qui viserait à explorer le plus finement possible l'intérieur de la «boîte noire » que constitue encore la classe, je m'intéresse plus particulièrement à la relation entre les enseignant-e-s et les élèves, telle qu'elle est vécue et construite au jour le jour par l'ensemble des protagonistes. Que savons-nous de ce qui se joue à chaque instant en cours, de la façon dont enseignant-e-s et élèves s'accordent au quotidien pour parvenir à «tenir » ensemble et, si possible, définir et mener à bien un projet commun d'apprentissage ? Que savons-nous de leurs rites, leurs codes, leurs valeurs, et de la mise en place de pratiques acceptables et acceptées par tous et toutes? Comment se négocient les compromis?

Mal, serait-on tenté de dire. On sait bien que les débats véritablement instituants se font rares. Quand bien même ils existeraient (et ils existent, au moins dans certaines classes d'inspiration Freinet ou de pédagogie institutionnelle), cela n'y suffirait pas. Car ce ne sont pas seulement les grandes institutions de la classe qui doivent se négocier, mais bien les micro-institutions permanentes, le droit de prendre la parole, le devoir d'écouter, l'importance donnée à telle parole plutôt qu'à telle autre, le statut de tel acte de travail, les regards, les sourires, les intonations, qui sont, en permanence à définir. Tous ces codes, ces savoirs du métier d'élève et d'enseignant qui permettent à chacun et chacune de comprendre et négocier sa place, notamment en termes de rapport au savoir dans l'espace commun ainsi construit. 
Car ces métiers sont loin d'être définis. Comment le seraient-ils quand les instructions officielles sont elles-mêmes contradictoires ? La loi Jospin de 1989 place en effet « l'élève au centre du système éducatif », tout en lui imposant des programmes extrêmement détaillés à suivre, de préférence, en temps et en heure. Comment peut-on, en tant qu'enseignant-e, être attentif à chaque élève, tout en respectant l'ensemble des prescriptions auxquelles on est soumis-e ? De l'idéal de Jules Ferry (que chaque jeune Français-e d'une classe d'âge donnée fasse la même dictée à la même heure) aux pédagogies dites «innovantes », la contradiction est loin d'être résolue. Quand le texte libre doit être "pour demain et si possible sur l'automne » comme le dit si bien B. Charlot (1993), ou que la « découverte » de l'élève tombe invariablement sur la partie prévue du fameux programme comme l'analyse M.-C. Baïetto (1982), il faut questionner plus avant les injonctions paradoxales d'une institution qui mettrait ses protagonistes en sérieux danger psychique s'ils et elles ne développaient pas de solides défenses.... De P. Ranjard (1984 et 1997) à C. Blanchard-Laville (2001), nombreux/ses sont les auteur-e-s qui ont montré les positions difficilement tenables dans lesquelles se trouvaient les enseignants et les solutions de compromis, avec eux-mêmes et avec l'institution, mises en place. Il reste donc aujourd'hui à voir comment ces compromis personnels s'articulent avec ceux des élèves, l'ensemble des protagonistes étant pris dans des contraintes aussi bien internes qu'externes ${ }^{1}$. Comment les enseignant et les enseignantes font-ils et elles au quotidien pour décider de leur action ? Quels effets leurs pratiques ont-elles sur les élèves ?

Pour approcher le plus finement possible les vécus et les ressentis, plusieurs techniques de recueil de données (entretiens, observation directe ou indirecte, etc.) peuvent être utilisées. Mais, si l'on veut saisir le sens des pratiques et des discours à la fois pour la micro-société que constitue la classe et pour les individus, on gagne, quel que soit le matériel, à articuler pour l'analyse une double grille, anthropologique et clinique à orientation psychanalytique. L'anthropologie permet de construire le cadre, la clinique à orientation psychanalytique contribue à l'analyse de la subjectivité de chacun-e en la replaçant dans ce cadre ; l'ensemble donne quelques pistes de compréhension générale du fonctionnement des un-e-s et des autres en situation pédagogique. Je vais tâcher d'en donner ici quelques exemples via une relecture de mes travaux, illustrée de ma propre pratique d'enseignante universitaire.

\section{L'idéal de la relation pédagogique : entre espace transitionnel et mimesis}

J'ai commencé par soutenir une thèse d'orientation clinique où j'interviewais des élèves et des enseignant-e-s engagé-e-s dans une pratique volontaire des mathématiques en-dehors de la classe (Hatchuel, 1997a) ${ }^{2}$. J'y montrais la nécessité, ou tout au moins l'utilité, pour pouvoir s'approprier du savoir, d'un espace potentiel, au sens de Winnicott (1975 et 1978), permettant de faire le lien entre soi et le savoir, c'est-à-dire d'un espace où l'élève puisse expérimenter et se tromper sans risque psychique, dans des directions qu'il ou elle aura librement choisies. Il m'apparait aujourd'hui, en retravaillant cette notion, que l'espace potentiel que l'enseignant-e va contribuer à créer pour chaque élève, en lui offrant une garantie minimale de prise en compte de ce qu'il ou elle est, est bien ce qui permet à l'élève, de là où il ou elle est, de partir affronter le monde du savoir, de la même façon que le jeune enfant peut investir le monde extérieur à partir de et en fonction de ce que l'adulte crée autour de lui. G. Mendel (1999) utilise à cet égard l'image très parlante d'Athéna et Ulysse : Athéna dit à Ulysse la réalité du monde extérieur et des dangers qu'il lui faudra affronter, mais elle fait confiance à son ingéniosité et à sa capacité à trouver par lui-même et pour lui-même comment affronter ces dangers. C'est donc bien lui qui construit son propre chemin, en fonction de ce qu'il est, ce qu'il souhaite et de ce qui lui convient, et qui peut donc en tirer fierté et profit, mais elle

\footnotetext{
${ }^{1}$ Sur la notion de contraintes internes, voir C. Blanchard-Laville, op. cit.

${ }^{2}$ La partie concernant les élèves a donné lieu à un article (Hatchuel, 1999a) et un livre (Hatchuel 2000a), tandis que celle concernant les enseignant-e-s a été publiée dans un ouvrage collectif (Hatchuel, 2000b)
} 
l'empêche de s'illusionner et le prévient de ce qu'il n'aurait pu percevoir seul. Enfin, elle intervient dans les rares cas où Ulysse ne pourrait réellement pas s'en sortir seul. Elle joue donc le rôle de filtre entre Ulysse et la réalité, mais un filtre non masquant qui, au contraire aide Ulysse à acquérir une pleine conscience de cette réalité en sélectionnant pour lui les éléments les plus marquants. Si on applique cette image au couple enseignant/ élève, on voit donc que le rôle de l'enseignant-e serait de présenter à l'élève la réalité du savoir à acquérir, et le caractère incontournable de cette acquisition (assez bien symbolisé par le programme) tout en lui laissant une marge de manœuvre suffisante pour pouvoir investir, c'est-à-dire amener dans son monde intérieur, ce savoir a priori extérieur à lui-même. Les processus d'investissements sont longs et complexes, ils demandent à être accompagnés au plus juste, en laissant l'élève se débrouiller seul-e tant qu'il/elle le peut, tout en intervenant, avant tout pour encourager, éventuellement pour (ré)orienter le travail, et en dernier ressort seulement pour (re)faire à la place de. Peuvent alors se mettre en oeuvre ce que C. Wulf (1999) appelle des «processus mimétiques », à partir du concept de mimesis (ou imitation créatrice) d'Aristote. Dans les processus mimétiques, l'enfant répète et imite mais, ce faisant, et parce que ce qui lui est proposé n'est pas trop fortement prescrit, il saisit peu à peu le sens de ce qu'il fait et l'adapte à ses propres projets.

Une telle attitude est évidemment un idéal à atteindre. Elle demande à l'enseignant-e d'être à peu près au clair sur ses propres enjeux afin qu'ils ne parasitent pas trop la rencontre pédagogique et laissent une souplesse de réaction et d'adaptation à la réalité de l'élève. C'est un travail permanent et jamais définitivement acquis d'élaboration de nos réactions d'enseignant-e-s dont C. Blanchard-Laville (op. cit.) témoigne à partir des groupes d'analyse de pratique d'inspiration Balint qu'elle anime et des entretiens et enregistrement de séances de cours sur lesquels elle travaille depuis 20 ans.

Dans la réalité, nous avons bien du mal à délier nos propres enjeux du travail de l'élève. Nous nous énervons face à un élève trop lent parce que nous ne supportons pas notre propre lenteur, nous attendons un exercice parfait pour être rassuré-e-s sur notre perfection d'enseignant-e, nous voudrions que l'apprentissage soit plus facile pour ne pas avoir à confronter l'autre à de la difficulté, etc. Se mettent alors en place ce que j'ai appelé des «couples psychiques épistémiques », c'est-à-dire des rencontres fortes entre un-e enseignant-e et un-e élève autour du savoir parce que l'enseignant-e tient à l'élève, à travers la façon dont il le met et se met en scène, un discours inconscient sur le savoir qui va résonner avec ce que l'élève a envie d'entendre. Or, selon que ce discours conforte l'élève dans ses illusions, qu'il lui montre un chemin plus ou moins balisé, ou plus ou moins encourageant, il va l'aider ou non à s'engager dans cet espace (voir Hatchuel, 1999a et 2000a).

\section{La mise en place d'une démarche d'ethnographie clinique : l'étude d'un exemple d'interactions élèves/ enseignante}

Les entretiens d'élèves nous disent donc que ce sont avant tout nos pratiques, au quotidien, qui sont ou non encourageantes, et qui vont permettre, via la rencontre avec l'enseignant-e, la rencontre avec le savoir. J'ai alors voulu aller plus avant dans ce travail de compréhension de l'interaction, pour saisir in situ les compromis qui pouvaient se mettre en place. J'ai pu, pour cela, mettre à profit le travail réalisé au sein d'une équipe dirigée par C. Blanchard-Laville dans une perspective que nous avons qualifiée de «codisciplinaire » sur des enregistrements vidéos de leçons de mathématiques.

Le travail codisciplinaire (voir Blanchard-Laville 2000) se donne pour objectif d'approcher, via des paradigmes disciplinaires différents, ce matériel commun et de confronter ces différents points de vue. Deux ouvrages (Blanchard-Laville, 1997 et 2002) sont issus de ce travail. Dans le premier, qui portait sur une leçon de numération sur les grands nombres au CM1, j'ai adopté une démarche que l'on peut qualifier d'ethnographique puisqu'elle consiste 
à tenter de saisir le point de vue de chaque acteur ou actrice à partir de son comportement et de ses interactions avec l'entourage. Ceci m'a amenée à établir, pour chaque élève, une fiche regroupant l'ensemble des informations le concernant (voir Hatchuel, 1997b). J'ai commencé par retranscrire les interactions avec l'enseignante (celles entre élèves ne sont malheureusement pas disponibles) et leur minutage (durée et répartition dans la séquence). Dans une perspective clinique, j'ai ensuite fait résonner en moi chaque dialogue comme si je le vivais du point de vue de l'élève, afin de m'approcher de ce que pourrait être le ressenti de chacun-e lors de cette séance. Certains points communs me sont alors apparus dans ce ressenti, que j'ai complétés par les positions socio-scolaires des élèves (profession des parents et résultats aux tests trimestriels de mathématiques) pour aboutir à un regroupement des élèves en sept catégories, auxquelles j'ai volontairement donné des qualificatifs qui exprimaient l'aspect subjectif de cette construction.

Sur le plan méthodologique, il s'agit donc non pas d'administrer une quelconque " preuve », mais de faire ressortir un point de vue identificatoire avec les élèves : qu'est-ce que j'aurais personnellement ressenti si je m'étais trouvée placée dans une telle situation ? Comment me serais-je située ? En cohérence avec une démarche clinique (voir par exemple BlanchardLaville 1999), l'objectif est d'analyser et de mettre en mots mes propres mouvements transférentiels afin de donner du sens (un sens faudrait-il même dire) à une situation. On aboutit alors à une typologie dont la valeur est avant tout heuristique et vise à construire non pas des certitudes qui décriraient l'alpha et l'oméga d'une situation mais ce que j'appelle un « savoir de vigilance » : parfois, dans certaines classes, il se peut que des élèves ressentent ce que je décris et il peut être utile d'y prêter attention.

Les deux élèves les plus "visibles" de cette typographie sont deux garçons occupent le devant de la scène en quasi-permanence (le temps maximum durant lequel ils peuvent rester silencieux est de 10 minutes) et font en quelque sorte leur « show ». Je les ai donc qualifiés de " showmen ", avant de constater qu'ils étaient tous deux en position socio-scolaire haute. En quasi miroir (dans la même position socio-scolaire mais différentes par le sexe), deux fillettes se caractérisent par une «tenue » (dans tous les sens du terme) impeccable, n'intervenant que quand on les interroge, et toujours pour rappeler la mémoire didactique de la classe ; elles sont, pour moi, des «petites filles sages ». Sept élèves en position socio-scolaire moyenne voire médiocre se voient très souvent ignoré-e-s ou rejeté-e-s lorsqu'ils et elles demandent la parole : ceci m'a amenée à les qualifier de « déclassé-e-s ». Je considère comme « repêché-e-s » des élèves timides que l'enseignante sollicite en fin de séance, une fois que l'essentiel des notions du jour semble acquis, et qui seraient, sans cela, resté-e-s silencieux ou silencieuses. Une élève en assez grande difficulté est interrogée à plusieurs reprises afin de vérifier son niveau de compréhension et de réguler la progression de la classe ; en cela, elle rend largement service à l'enseignante et à la classe ; j'ai donc employé, la concernant, l'expression imagée de « nulle de service ", avant d'estimer que quatre autres élèves pouvaient plus ou moins se ranger dans cette catégorie (avec des nuances et des hésitations que je ne développerai pas ici). Enfin, cinq élèves n'entretiennent quasiment aucune interaction avec l'enseignante: je les ai donc qualifié-e-s d'« inexistant-e-s ».

Etant donné leur mode de construction, ces catégories sont donc évidemment à prendre comme des tendances, des indications, non des modes de fonctionnement rigides dont il faudrait accuser l'enseignante. Mais elles permettent de percevoir sur quel mode l'enseignante tend à se positionner face à chaque élève, et quels peuvent être quelques-uns des principes organisateurs de sa relation aux élèves (repousser ceux et celles qui la sollicitent, à quelques exceptions près, solliciter les silencieux et silencieuses, etc.). Restait alors à savoir dans quelle mesure ces catégories étaient «transposables» et quel était leur degré de contingence. Les mêmes élèves, avec une même enseignante et avec des enseignant-e-s différent-e-s, se retrouvent-ils et elles toujours dans la même catégorie ? Comment passe-t-on de l'une à l'autre, 
comment se construit une identité d'élève et, par là, un rapport au savoir ? C'est dans cet esprit que j'avais abordé le travail suivant.

\section{La troisième étude : une réflexion autour d'éventuels invariants anthropologiques}

Le plus flagrant dans la deuxième leçon étudiée (un cours sur la multiplication des fractions en $5^{\mathrm{e}}$ ) réside dans la différence de climat par rapport à la classe de CM1. La où les élèves de CM1 allaient et venaient au tableau, en un ballet effervescent les y amenant parfois à trois ou quatre, parfois même sans que l'enseignante ne s'en aperçoive immédiatement, les $5^{\mathrm{e}}$ sont beaucoup plus soumis-e-s à un enseignant-e qui leur laisse une marge de manœuvre extrêmement réduite. Leur activité est soigneusement contrôlée dans ses moindres détails, qu'il s'agisse de prendre le bon cahier, d'ouvrir la calculatrice au moment opportun et pas avant, de choisir la couleur de la craie utilisée ou de répondre au mot près la phrase attendue. Très souvent, l'enseignant utilise une élève dans une fonction que je qualifie de « porte-craie », consistant à écrire sous sa dictée sans dire un mot. Enfin, nous avons tou-te-s été frappé-e-s, et c'est ce qui nous avait fait choisir cette séquence, par un épisode un peu long (par rapport aux temps très courts que les autres élèves passent au tableau) entre l'enseignant et une élève que nous avons prénommée Mélanie, au point que nous l'avons choisie pour le titre du livre issu de ce travail (voir Blanchard-Laville, 2002). Cette élève est appelée au tableau en fin de séance, pour corriger une multiplication de quatre fractions non simplifiées, soit un exercice relativement difficile. Certes, il ne s'agit que d'une extension de deux règles précédemment apprises (multiplication de deux fractions simplifiées, vue en début d'heure, et simplification usuelle des fractions). Mais on sait bien qu'un tel travail de transposition est loin d'être simple, et l'élève précédant Mélanie au tableau, considéré comme le meilleur élève de la classe, sur un exercice similaire, avait disposé d'un minimum de temps et de marge de manœuvre pour réfléchir et intégrer la nouvelle situation. C'est le seul qui se voit offrir ce qui peut être considéré comme un luxe durant cette leçon.

Mélanie, elle, n'a pas cette chance. Très vite, elle est tournée en dérision («tu as perdu ta langue ?») et stigmatisée pour sa lenteur. Il faut dire qu'elle manifeste une mauvaise volonté évidente, prenant quasiment l'enseignant à son propre piège : puisque tout est prescrit, elle attend tranquillement les instructions qu'elle exécute très fidèlement. Mais, et c'est là la perversité du système, les instructions présupposent un minimum d'initiatives de sa part, étant entendu que ces initiatives doivent coller aux attentes de l'enseignant. "Fais ce que tu as faire » lui dit-il par exemple, alors qu'elle vient de proposer de regrouper les fractions par deux, pour se retrouver dans une situation connue, et qu'elle en a été empêchée. "Je ne comprends pas, monsieur» répond Mélanie, prise au piège d'une injonction magnifiquement contradictoire. Il s'avère alors bien difficile de démêler la situation. Mélanie, qualifiée par ailleurs de «décevante » par l'enseignant, et qui montre ostensiblement, depuis le début de la leçon, son désintérêt pour ce qui se passe, ne prend-elle pas d'initiatives parce qu'elle sait (ou pense) que ses initiatives seront rejetées, ou ses initiatives sont-elles rejetées parce qu'elles ne sont pas adaptées et dénotent de façon évidente sa non-implication dans la situation? Les deux vérités sont bien entendu acceptables, selon le point de vue où l'on se place, et c'est un des grand enjeux de la recherche que de montrer comment ces «mondes vécus » ${ }^{3}$ s'acceptent et cohabitent.

Toujours est-il, que les catégories précédemment évoquées ne se retrouvent pas : l'espace étant envahi par l'enseignant, on ne retrouve, logiquement, pas de « showman »; aucune des filles de la classe ne semble être traitée différentiellement pour sa capacité à rappeler le savoir acquis ; l'enseignant ne semble pas particulièrement attentif à certain-e-s élèves et si certain-e-s élèves sont particulièrement stigmatisé-e-s, on ne peut dire ni qu'il s'agit des plus faibles de la classe, ni que les plus faibles sont utilisé-e-s pour réguler l'avancée du cours en fonction de leur compréhension. La compréhension des élèves n'est de toutes façons jamais vérifiée dans le but de procéder éventuellement à une remédiation, mais le plus souvent dans une perspective

\footnotetext{
${ }^{3}$ Au sens de J. Habermas (voir par exemple Cusset, 2001).
} 
d'accusation de l'élève qui n'a pas compris. Finalement, il n'y a guère que la catégorie des « inexistant-e-s » et, dans une moindre mesure, celle des «déclassé-e-s » que l'on retrouve vraiment dans les deux cas.

Mais ce que l'on retrouve, par contre, c'est ce que l'on pourrait qualifier de " métacatégories », de regroupement de catégories. Qu'il s'agisse de faire son show ou plus simplement de se donner de la place pour penser, qu'il s'agisse de répéter le savoir ou de ne pouvoir progresser, la question est bien toujours de savoir quelle place est réservée à et se réserve l'élève, quel rôle l'enseignant-e lui fait et il accepte de jouer et comment, de cette place-là et avec ce rôle-là il ou elle peut ou non entrer dans l'apprentissage.

S'il est évident que tout-e enseignant-e traite différentiellement ses élèves, il apparaît donc que les formes prises par cette différentiation dépendent de la situation-classe et des caractéristiques personnelles de l'enseignant-e : il y a, dans la leçon sur les grands nombres des « déclassé-e-s » et des « repêché-e-s » parce que l'enseignante déclasse et repêche, et dans celle sur les fractions des «souffre-douleur» et des «porte-craies », parce que ce sont les modalités particulières d'interaction de cet enseignant. Mais, au-delà de ces contingences, il s'agit bien toujours d'accepter ou de rejeter, d'aider ou de permettre, d'encourager ou d'ignorer, c'est-à-dire de modalités d'incarnation, en situation, des archétypes anthropologiques de l'entrée en relation que sont la peur, l'aide, l'encouragement, le conflit, l'ignorance, la domination, etc. Les catégories précédentes seraient alors des modalités contingentes d'expression de ces métacatégories, que l'on pourrait également aborder en termes psychanalytiques: quel espace chaque mode de relation réserve-t-il à l'élève, et aux différentes instances de son psychisme (Moi Idéal/ Surmoi/ Idéal du Moi)?

\section{La régulation de l'échange : l'exemple de notre groupe de travail}

C'est à partir de l'étude des institutions (au sens de la pédagogie institutionnelle) internes à notre groupe de recherche codisciplinaire que j'ai pu développer ce point de vue. Nous avons, en effet, essayé, tout au long du travail, d'être particulièrement vigilant-e-s à ce qui rendait possible la rencontre codisciplinaire et une véritable appréhension par chacun-e du travail de l'autre. C. Blanchard-Laville (2000) a développé l'importance du dispositif d'élaboration collective tandis que je m'attachais à approfondir, à partir de la question de savoir ce que représentait un texte écrit, donné, lu et commenté les notions d'échange, de don et de dette dans le cadre du travail intellectuel. Qu'est-ce que je donne (et donc qu'est-ce que j'attends en retour) et qu'est-ce que je reçois lorsque j'écoute ou lis l'autre ? Lorsque je produis pour un groupe ? Lorsque je m'intéresse au travail de l'autre ? Etc. A quelles conditions chacun-e peut-il ou elle se sentir satisfait-e et non lésé-e du fonctionnement collectif et va-t-il ou elle pouvoir se construire dans cet échange ${ }^{4}$ ? Dans cette problématique où les catégories classiques de l'anthropologie s'appliquent au savoir, les questions du rythme et de l'attente apparaissent comme essentielles. Quel temps laissons-nous à l'autre pour s'approprier nos travaux, et se repositionner en fonction de son propre passé, de notre passé commun et de cette nouveauté ? Quelle attente comble et crée chaque nouveau texte ? Comment accepter que l'autre ne travaille pas à mon rythme ? Trop lent-e, j'ai le sentiment d'être seul-e à faire avancer le collectif, trop rapide, il ou elle m'étouffe de trop de nouveauté (voir Hatchuel, 1999b).

Il m'a semblé alors que l'on pouvait appliquer cette grille d'analyse aux relations enseignante-s/ élèves. De la codisciplinarité, on ne peut à proprement parler garder ici l'aspect « discipline » puisque les élèves ne sont pas porteurs de paradigmes conceptuels précis, mais la notion de «cotravail» ou de «copensée » telle que peut, par exemple, la définir D. Widlöcher (1994) peut nous aider à questionner la rencontre pédagogique. Je propose alors de

\footnotetext{
${ }^{4}$ A partir de l'idée que c'est à travers la relation de dette, de don et de contre-don, que se forment ou se déforment les identités (Godbout, 2000)
} 
le faire à partir de la notion de confiance : qu'est-ce qui fonde la confiance entre enseignant-es et élèves ? Pour quoi et pourquoi vais-je faire confiance à l'autre ? Quels sont les signes que chacun-e prélève pour établir ou retirer sa confiance, en fonction des efforts préalablement fournis et donc des retours attendus ? A partir de quel moment va-t-on estimer que chacun-e a rempli sa part du travail commun, et payé le coût, psychique et symbolique, du bénéfice attendu ? A défaut de parole, c'est à travers l'infinitésimal des signaux codés que ces questions s'élaborent. On aborde là la notion de culture, qui dépasse bien évidemment largement celle d'ethnie, voire de groupe social. On pourrait, à la limite, donner une définition au moins partielle de la culture comme constituée justement de cet ensemble de signaux et de codes permettant à chacun-e de réajuster en permanence attentes et efforts, ce qui ne serait pas très éloigné de l'habitus bourdieusien (Bourdieu, 2000). On imagine bien que ce réajustement est rarement d'une adéquation parfaite surtout pour les élèves les plus en difficulté. Or ce sont bien ces élèves-là qui ont le plus de mal, on le sait, à maîtriser les codes de l'école et donc à trouver leur place, à exister comme individu à l'école puisque, finalement, on peut considérer un code comme un savoir intégratif qui permet de comprendre et négocier sa place dans le monde ${ }^{5}$. Il importe donc d'aider les élèves, et surtout ceux et celles en difficulté, à exister pour ce qu'ils et elles sont dans l'espace de la classe. Ce qui peut passer par le fait de leur laisser, d'une façon ou d'une autre, des possibilités pour réagir à ce qui leur est proposé, dans un cadre permettant de garantir que cette réaction, si elle peut être imprévue et surprenante, ne saurait être inadéquate ou inadmissible.

\section{Un indicateur de la régulation : la notion de temps de latence}

Une de ces possibilités, qui me semble en même temps constituer un indicateur pour la recherche, réside dans la notion de temps de latence, c'est-à-dire ces micro-temps que l'enseignant-e laisse (ou ne laisse pas) entre deux questions, deux prescriptions, deux indications. On retrouve, à une échelle plus petite adaptée au travail des élèves, l'importance de la temporalité évoquée à propos de notre équipe. Dans la classe de $5^{\mathrm{e}}$, par exemple, Mélanie ne dispose d'absolument aucun temps de latence : tout ce qu'elle fait (ou ne fait pas) est immédiatement commenté, décortiqué, critiqué, sans qu'elle-même puisse jamais rien en dire. Cette notion me semble une des clés pour comprendre ce qui, dans les rituels (dont $\mathrm{C}$. Wulf (op. cit.) dit qu'ils sont le support de la mimesis) rend justement possible cette imitation créatrice : si le rituel garantit le cadre, et donc une certaine adéquation, il doit également permettre la création personnelle. Et c'est peut-être lorsqu'ils laissent des temps de latence, lorsqu'ils sont en quelque sorte "étirés » que les rituels deviennent des supports de la créativité et donc de la construction de soi de chacun-e. Les rituels ne peuvent permettre d'imitation créatrice que si, à un moment, ils sont mis en place par nous-mêmes, appropriés. C'est un jeu subtil autour de « je transforme en m'appropriant, je m'approprie parce que je transforme, je transforme parce que je m'approprie » que l'on peut rapprocher de la notion d'acte-pouvoir de G. Mendel (1985) : on perçoit et récupère le pouvoir de ses actes à condition d'avoir du pouvoir sur ses actes. On pourrait donc parler de rituel exogène ou hétéronome (qui vient de l'extérieur) et de rituel endogène, autocentré, qui vient de soi ou, dans un autre registre, de rituels oppressifs opposés aux rituel créatifs. Un rituel créatif peut finalement être considéré comme le résultat d'un compromis, et il se recompose en

\footnotetext{
5 J'avais été particulièrement touchée, parmi les élèves que j'avais interviewé-e-s pour mon doctorat, par la souffrance d'Aline, alors âgée de 26 ans, qui, après un parcours scolaire chaotique mais se terminant finalement par un BTS, peinait à trouver du travail et évoquait son incompréhension face à ce monde de l'entreprise qu'elle ne comprenait pas. Elle découvrait alors à quel point les codes de l'école, qu'elle avait eu tant de mal à s'approprier, ne pouvaient lui suffire. Ne sachant pas comment se comporter au quotidien, elle se trouvait extrêmement désemparée, désarroi qui la renvoyait probablement à ses difficultés d'élèves alors même qu'elle pensait les avoir surmontées.
} 
permanence en fonction des circonstances tout en s'inscrivant dans l'histoire. Un rituel est propre à un groupe à un instant $\mathrm{t}$, tout en étant porteur du passé des individus et du groupe. Parce qu'il se transforme, mais lentement, il permet de constituer et tenir le cadre tout en laissant chacun-e trouver sa place.

\section{Contribuer à construire des rituels créatifs : des espaces d'élaboration}

Les conditions de construction et de transformation des rituels (qui incluent les institutions au sens de la pédagogie institutionnelle mais ne s'y réduisent pas) apparaissent alors comme essentielles. On peut les laisser agir au quotidien, à condition comme on l'a vu, de ne pas trop les «resserrer », mais il faut également souligner l'intérêt et la pertinence d'espaces d'élaboration et/ou de parole où chacun-e puisse apprendre sans danger et/ou comprendre quelque chose de ce qu'il ou elle peut attendre de l'autre et ce qu'il ou elle peut lui offrir en retour. Car de tels espaces d'élaboration individuelle et/ou collective permettent de favoriser une véritable prise en compte de l'autre et une rencontre respectueuse des différences et consciente de leur irréductibilité. Il s'agit, en fin de compte, d'accepter l'idée que l'autre ne fera pas forcément ce que l'on croyait légitimement pouvoir être en droit d'attendre de lui ou d'elle. Un tel travail n'est pas simple, il demande du temps (là encore) et des dispositifs adéquats. Selon le niveau auquel on se place et le point de vue que l'on privilégie, on pourra penser à des pratiques diverses : les groupes d'analyse de pratique d'inspiration Balint tels que les anime C. Blanchard-Laville (2000), centrés sur l'élaboration individuelle de chaque participant-e, contribuent à délier les répétitions à l'œuvre chez chaque inidividu ; les groupes de parole d'étudiant-e-s que j'ai mis en place en année préparatoire à la licence de sciences de l'éducation à Paris $X$, aident ces étudiant-e-s en reprise d'études (donc souvent avec un rapport complexe à l'idée même de reprendre un travail universitaire) à se définir et se situer comme étudiant-e-s, à l'université mais aussi dans le reste de leur vie; le dispositif d'expression collective des élèves mis en place par des conseiller et conseillères d'orientation psychologue à partir des travaux de G. Mendel (voir Rueff 1997) permet à un collectif d'élèves de construire un minimum d'accord sur ce qu'il souhaite transmettre aux adultes qui le prennent en charge et aux adultes d'y répondre également de façon collective ; les stages de formation continue d'enseignant-e-s dits «sur site» (cf. Hans, 1999), c'est-à-dire dans l'établissement même, amènent les enseignant-e-s à travailler ensemble, à travers des questions comme l'autorité, la loi, etc., autour de leur identité, de leurs statuts et de leurs rôles. Mais il faut penser également, même s'ils peuvent sembler très éloignés de ces dispositifs tous plus ou moins inspirés de la psychanalyse et /ou de la psychologie sociale clinique, à tous les lieux de débat et de confrontation des statuts et rôles de chacun-e, pourvu que les conditions soient requises (ce qui ne va pas de soi) pour que les demandes et les attentes n'apparaissent pas comme des exigences. Il peut s'agir de lieux aussi variés que des rencontres avec les parents d'élèves, des conseils de classe ou d'administration, des concertations enseignantes, des rencontres militantes ou syndicales, voire des inspections ou des débats nationaux autour du système scolaire.

Car ce qui me semble important, à chaque niveau d'accord avec soi-même ou avec les autres, collègues, partenaires, hiérarchie ou élèves, c'est de pouvoir dire quelque chose de cet accord, de ce qu'on prend en charge et ce que l'on considère comme étant du ressort de l'élève, ce qui est obligatoire et ce qui ne l'est pas. Les étudiant-e-s auxquel-le-s j'enseigne savent qu'ils et elles doivent fournir un travail de qualité. J'essaye de définir le plus précisément possible ce qu'est, pour moi, un travail de qualité en termes de questionnement, de rigueur, de clarté ou de recul, de leur faire s'approprier ces critères (par exemple en travaillant sur des travaux des années précédentes) mais ensuite ils et elles ont toute liberté pour choisir le thème et s'organiser. Dans le même temps, je sais que si je ne suis pas attentive aux plus timides et aux moins réalistes, cette liberté risque de se retourner contre eux, comme le rapporte si bien 
S. Boimare (1999) à travers les propos d'un adolescent pour qui « des fois les parents ils nous donnent exprès des choses trop difficiles pour qu'on se trompe ». Le plus difficile est alors de savoir quand relancer, fixer des ultimatums, donner des conseils et avec quel degré d'ouverture ou au contraire de fermeté, etc. C'est bien là que se construisent les rituels, suffisamment cadrant pour (tenter de) garantir que le travail sera fait, suffisamment ouverts pour permettre que ce qui sera fait appartiendra réellement aux étudiant-e-s.

\section{En conclusion : vers une culture de la responsabilité ?}

Nous pouvons alors revenir à notre question de départ («quelle place laissée (laisser ?) à l'élève face au programme ? ») et y apporter un début de réponse en termes d'autonomie « raisonnable». : une autonomie «raisonnable», ce serait celle que l'on peut assumer. En effet, toute liberté implique la responsabilité des choix qu'elle engendre, et si nous faisons, chacun-e à notre niveau, comme nous pouvons, il ne saurait être question de nous désolidariser des conséquences de nos actes. Définir ce qui est de la liberté (et donc de la responsabilité) de chacun-e, enfant, jeune ou adulte, constitue, à mon avis, une bonne part de la réflexion collective afin que chacun-e puisse trouver comment, avec ses ressources propres, il ou elle peut avancer dans le sens qui lui semble le plus souhaitable. Il s'agit donc pour chacun-e de conquérir davantage d'autonomie c'est-à-dire non seulement davantage de liberté (et là, la conquête se fait sur l'extérieur) mais aussi (ce qui est une conquête sur soi) davantage de capacité à gérer cette liberté. Le combat éducatif et aussi, et avant tout, un combat politique.

\section{Bibliographie}

Baïetto, Marie-Claude (1982) Le désir d'enseigner. Paris, ESF.

Boimare, Serge (1999) L'enfant et la peur d'apprendre. Paris, Dunod.

Bourdieu, Pierre (2000) Esquisse d'une théorie de la pratique. Paris, Seuil. $1^{\text {ère }}$ éd. 1972.

Blanchard-Laville, Claudine (1997) Variations sur une leçon de mathématiques. Analyses d'une séquence : " L'écriture des grands nombres ». Paris, L'Harmattan.

Blanchard-Laville, Claudine (1999) «L'approche clinique d'inspiration psychanalytique : enjeux théoriques et méthodologiques »-Revue Française de Pédagogie 127 (9-22).

Blanchard-Laville, Claudine (2000) «De la codisciplinarité en sciences de l'éducation »Revue Française de Pédagogie 132 (55-66).

Blanchard-Laville, Claudine (2001) Les enseignants entre plaisir et souffrance. Paris, PUF.

Blanchard-Laville, Claudine (éd). (2002, à paraître) "Mélanie, tiens, passe au tableau». L'ordinaire d'une classe à l'aune de la codisciplinarité.

Charlot, Bernard (1993) «L'innovation n'est plus ce qu'elle était »-Autrement 136 (20-27).

Cusset, Yves (2001) Habermas, l'espoir de la discussion. Paris, Michalon.

Godbout, Jacques (2000) Le don, la dette et l'identité. Paris, La Découverte.

Hans, Danielle (1999) «Quelques réflexions sur les liens intersubjectifs dans l'établissement scolaire aujourd'hui : entre reproduction et changement »- in : L'innovation, levier de changement dans l'institution éducative, Actes des Universités d'été de la Baume-les-Aix et de Rennes. Ministère de l'Education Nationale, de la Recherche et de la Technologie.

Hatchuel, Françoise (1997a) Elèves et enseignant(e)s engagé(e)s dans une pratique volontaire des mathématiques: rapport au Savoir et processus identitaires. Etude clinique d'une innovation. Thèse de doctorat (dir. C.Blanchard-Laville), Université Paris X.

Hatchuel, Françoise (1997b) «"Dis, maîtresse, comment tu me vois ?". Assignation et étiquetage en situation d'enseignement collectif»-in : Blanchard-Laville C. (éd) Variations sur une leçon de mathématiques. Analyses d'une séquence : "L'écriture des grands nombres ». Paris, L'Harmattan. 
Hatchuel, Françoise (1999a) «La construction du rapport au Savoir chez les élèves: processus socio-psychiques »- Revue Française de Pédagogie 127 (37-47).

Hatchuel, Françoise (1999b) «Regard anthropologique sur la notion de codisciplinarité »in : Blanchard-Laville C. (éd.) Approches codisciplinaires des pratiques enseignantes dans leurs rapports aux apprentissages différentiels des élèves. Rapport final au CNCRE (une patie de cet article est repris dans Blanchard-Laville, 2002).

Hatchuel, Françoise (2000a) Apprendre à aimer les mathématiques. Conditions socioinstitutionnelles et élaboration psychique dans les ateliers mathématiques. Paris, PUF.

Hatchuel, Françoise (2000b) «Enseignant/es et innovation : enjeux institutionnels et rapport au savoir » - in : Mosconi Nicole, Beillerot Jacky, Blanchard-Laville Claudine (éd) Formes et formation du rapport au savoir. Paris, L'Harmattan.

Mendel, Gérard (1985) La crise est politique, la politique est en crise: de l'autorité traditionnelle à l'actepouvoir autogestionnaire. Paris, Payot.

Mendel, Gérard (1999) Le vouloir de création. La Tour d'Aigues, Editions de l'Aube .

Mendel, Gérard (2002) Une histoire de l'autorité. Permanence et variations. Paris, La Découverte.

Ranjard, Patrice (1984) Les enseignants persécutés. Paris, R.Jauzé.

Ranjard, Patrice (1997) L'individualisme, un suicide culturel. Les enjeux de l'éducation. Paris, L'Harmattan, (Savoir et formation).

Rueff, Claire (1997) La démocratie dans l'école. Une pratique d'expression des élèves. Paris, Syros.

Widlöcher, Daniel (1994) Les nouvelles cartes de la psychanalyse. Paris, Odile Jacob.

Winnicott, D.W. (1975) Jeu et réalité : l'espace potentiel. Paris, Gallimard (Connaissance de l'inconscient).

Winnicott, D.W. (1978) L'enfant et le monde extérieur. Paris, Payot (Sciences de l'homme). 1ère édition anglaise 1957.

Wulf, Christoph (1999) Anthropologie de l'éducation. Paris, L'Harmattan. 